\title{
Curso de Capacitação para Cirurgiões-Dentistas no Atendimento à Pessoa com Deficiência e Necessidades Especiais do Estado do Rio Grande do Sul: Perfil do Cirurgião-Dentista, Trajetória no Serviço e Cobertura dos Municípios
}

Training Course for Dentists on the Assistance to Persons with Disabilities and Special Needs of the Rio Grande do Sul State: Dentist Profile, Trajectory in Service and Municipalities Coverage

Alexandre Baumgarten ${ }^{1}$, Ana Paula Scherer Monteiro ${ }^{2}$, Renata Córdova Petersen ${ }^{3}$

\begin{abstract}
Objective: To analyze the profile of the dentists who performed the 'Training Course for Dental Surgeons in Care for People with Disabilities and Special Needs of the Rio Grande do Sul State ', his journey in caring for these patients and the coverage of municipalities benefited with the program. Methods: Cross-sectional study of exploratory-descriptive character, performed by a questionnaire application in 126 dentists (86,9\% of the total) who works in primary care, in Rio Grande do Sul State, and that participated in one of the ten editions of the related course between 2000 and 2010. A databank was created with the collected information in SPSS statistical package.

Results: The majority of the dentists are woman, work in SUS and Private Clinics, work on SUS by means of statutory regime with a weekly working hours of 40 hours. 68 related to attend patient with special needs in SUS basic health units. The main reasons given for the no attend were: there was no incentive for managers to implement a program to care special patients and there was not auxiliary staff capacitated. Just 58 of the 496 municipalities of the Rio Grande do Sul have one or more dentists capacitated that attended the mentioned course. Conclusion: The results identified the dentists profile, its trajectory in the care of these patients and the municipalities covered by the program.
\end{abstract}

Keywords: Disabled Persons; Health Services Needs and Demand; Oral Health; Dental Staff.

\section{Resumo}

Objetivo: Analisar o perfil dos cirurgiões-dentistas que realizaram o 'Curso de Capacitação para Cirurgiões-Dentistas no Atendimento à Pessoa com Deficiência e Necessidades Especiais do Estado do Rio Grande do Sul', seu percurso no atendimento a esses pacientes e a cobertura dos municípios beneficiados com o programa. Materiais e Métodos: Estudo transversal de caráter exploratório-descritivo, realizado por meio da aplicação de questionário em 126 cirurgiões-dentistas(CDs) ( $86,9 \%$ do total) atuantes na atenção básica, no Estado do Rio Grande do Sul, e que participaram de uma das dez edições do referido curso entre 2000 e 2010. Foi criado um banco de dados com as informações coletadas no software estatístico SPSS. Resultados: A maior parte desses cirurgiões-dentistas são mulheres, possuem vínculo empregatício com o SUS e Clínicas/Consultórios Privados, trabalham no SUS por meio do regime estatutário com uma carga horária semanal de 40 horas. 68 afirmam atender pacientes especiais nas unidades básicas de saúde do SUS. Os principais motivos apontados para o não atendimento foram: não houve incentivo por parte dos gestores para a implantação de um programa para atendimento de pacientes especiais e não há equipe auxiliar capacitada. Apenas 58 dos 496 municípios do Rio Grande do Sul possuem um ou mais CDs capacitados que passaram pelo referido curso. Conclusão: Os resultados encontrados permitiram identificar o perfil dos CDS, sua trajetória no cuidado a esses pacientes e os municípios cobertos pelo programa.

Descritores: Pessoas com Deficiência; Necessidades e Demandas de Serviços de Saúde; Saúde Bucal; Recursos Humanos em Odontologia
${ }^{1}$ Programa de Pós-Graduação em Epidemiologia. Universidade Federal do Rio Grande do Sul. Especializando em Odontologia para Pacientes com Necessidades Especiais - ABORS

${ }^{2}$ Especialista em Saúde Coletiva. Especializando em Odontologia para Pacientes com Necessidades Especiais - ABORS

${ }^{3}$ Especialista em Ortodontia. Especializando em Odontologia para Pacientes com Necessidades Especiais - ABORS

Correspondência: Alexandre Baumgarten

Endereço: Programa de Pós-Graduação em Epidemiologia. Universidade Federal do Rio Grande do Sul. Rua Ramiro Barcelos, $2400-2^{\circ}$ andar - Porto Alegre, Rio Grande do Sul, Brasil - 90035-003

E-mail: a.baumgarten@hotmail.com

Data de Submissão: 02/01/2015

Data de Aceite: 10/06/2015

\section{Introdução}

Para a classe odontológica, tradicionalmente tem se definido pacientes com necessidades especiais(PNE), aquelas pessoas que tem alguma doença ou situação clínica que necessitem um atendimento odontológico diferenciado (CONSELHO FEDERAL DE ODONTOLOGIA, 2012). As razões das necessidades especiais são inúmeras podendo apresentar uma ou mais limitações, temporárias ou permanentes, de ordem mental, física, sensorial, emocional, de crescimento ou médica, que o impeça de ser submetido a uma situação odontológica convencional (LUCENA et al., 2013; SILVESTRE, 2007).

Estas condições especiais acarretam em uma maior prevalência de doenças bucais como cáries, perdas dentárias, problemas periodontais, hábitos parafuncionais e má-oclusões (LUCENA et al., 2013; PEREIRA et al. 2010; ASA, 2007) e ocorrem principalmente devido a um déficit na higiene oral gerada pelas limitações físicas e psíquicas(CINGANO et al., 2013), pelo tipo de dieta(TANAKA et al., 2009), pela dificuldade em mastigar e deglutir(SANTOS; NOGUEIRA, 2005) ou pelos diversos medicamentos que são ingeridos diariamente(SANTOS et al., 2010a; SANTOS et al., 2010b).

Em setembro de 2014 o Conselho Federal de Odontologia aponta, para o estado do Rio Grande do Sul, apenas 25 especialistas habilitados na especialidade de Odontologia para Pacientes com Necessidades Especiais dos 16.106 cirurgiões-dentistas (CD) inscritos(CONSELHO FEDERAL DE ODONTOLOGIA, 2014).

$\mathrm{Na}$ tentativa de suprir a carência desses especialistas, o Estado do Rio Grande do Sul, através da Escola de Saúde Pública (ESP) e da Fundação de Articulação e Desenvolvimento de Políticas 
Públicas para Pessoas Portadoras de Deficiência e de Altas Habilidades no Rio Grande do Sul (FADERS), implantou no ano de 2000 o curso de capacitação para cirurgiões-dentistas e auxiliares para atender pacientes com necessidades especiais nas unidades básicas de saúde de seus municípios.

O curso, que teve início no ano de 2000 , se desenvolve através de aulas teóricas, ministradas na ESP, e aulas práticas, desenvolvidas em uma das unidades da FADERS. A seleção de alunos se dá pelo vínculo à rede pública de serviços de saúde através de entrevistas e análise de currículo. Essa capacitação tem como proposta estimular atendimentos aos PNE nas unidades básicas dos municípios do estado do Rio Grande do Sul, através do aperfeiçoamento dos recursos humanos (CD e equipe auxiliar) encaminhados pelos municípios interessados.

Nesse contexto, o presente artigo tem como objetivo analisar, de forma descritiva, o perfil dos cirurgiões-dentistas que realizaram o 'Curso de Capacitação para Cirurgiões Dentistas no Atendimento à Pessoa com Deficiência e Necessidades Especiais do Estado do Rio Grande do Sul', seu percurso no atendimento a esses pacientes e a cobertura dos municípios beneficiados com o programa.

\section{Materiais e Métodos}

Foi conduzido um estudo transversal de caráter exploratóriodescritivo, realizado no Estado do Rio Grande do Sul entre os meses de maio de 2011 e abril de 2012.

O 'Curso de Capacitação para Cirurgiões Dentistas e Pessoal Auxiliar em Odontologia para Atendimento à Pessoa com Deficiência e Necessidades Especiais' foi realizado entre os anos de 2000 a 2010, tendo anualmente uma edição. A população foi constituída dos 145 cirurgiões-dentistas que participaram de uma das dez edições desse curso, sendo que 126 manifestaram interesse em participar da pesquisa e constituíram a amostra deste estudo, 3 não quiseram responder ao questionário e 16 não foram encontrados.

Para a coleta dos dados foi empregada a técnica de autorrelato por meio de um questionário estruturado(POLIT; BECK; HUNGLER, 2004), composto por três partes. Na primeira parte do questionário foram identificados os indivíduos e coletadas informações sobre o vínculo empregatício (serviço público e privado), além da carga horária. A segunda parte questionou sobre 0 atendimento a pacientes com alguma necessidade especial. Por fim a última parte buscou mapear o município em que o profissional exerce a capacitação.

Foi realizado contato telefônico, com todos os $C D$ que participaram do curso antes de enviar os questionários buscando esclarecer os objetivos da pesquisa. Após o questionário foi enviado por e-mail, juntamente com o Termo de Consentimento Livre e Esclarecido. As informações eram registradas pelos sujeitos, mas eles tinham a oportunidade de esclarecer dúvidas com 0 entrevistador.

Para a análise das questões objetivas do questionário foi criado um banco de dados digitado no software estatístico IBM SPSS Statistics versão 18.0. Os dados foram tabulados e posteriormente analisados por intermédio de procedimentos da estatística descritiva, mediante cálculo de frequência relativa das respostas emitidas às questões(TOBAR; YALOUR, 2001). O mapeamento dos municípios em que os profissionais exercem a capacitação foi realizado por meio da distribuição por mesorregiões do Rio Grande do Sul.

O estudo foi aprovado pelo Comitê de Ética em Pesquisa da Escola de Saúde Pública do Rio Grande do Sul (Projeto: 663/11) e todos participantes assinaram o termo de consentimento livre e esclarecido.

\section{Resultados}

De um total de 145 cirurgiões-dentistas que realizaram o curso de capacitação para atendimentos a pessoas com necessidades especiais entre 2000 e 2010, 126 responderam ao questionário (taxa de resposta de $86,9 \%$ ). Destes cirurgiões dentistas, a maioria eram mulheres $(74,6 \%)$, possuem vínculo empregatício com o Sistema Único de Saúde (28,7\%), seu vínculo com o SUS é através de regime estatutário $(61,1 \%)$ e possuem uma carga horária semanal de 40 horas no SUS(39,7\%) (Tabela 1).

Tabela 1 - Perfil do CD capacitados pelo curso no atendimento de PNE.

\begin{tabular}{llcc}
\hline & VARIÁVEIS & $\mathbf{n}$ & $\mathbf{\%}$ \\
\hline \multirow{2}{*}{ SEXO } & Feminino & 94 & 74,6 \\
& Masculino & 32 & 25,4 \\
& Somente Sistema Único de & 36 & 28,7 \\
VíNCULO & Saúde & 19 & 15,0 \\
EMPREGATícIO & Somente Serviço Privado & 61 & 48,4 \\
& Sistema Único de Saúde e & 10 & 7,9 \\
& Privado & 77 & 61,1 \\
& Não Exerce Mais Odontologia & 13 & 10,3 \\
VíNCULO COM OS & Estatutário & 3 & 2,4 \\
SUS & Celetista & 4 & 3,2 \\
& Estatutário e Celetista (2 & 29 & 23,0 \\
& concursos) & 4 & 3,2 \\
& Prestador de Serviço (Autônomo) & 4 & 1,6 \\
& Não Trabalha Mais no SUS & 34 & 27,0 \\
CARGA HORÁRIA & 10 & 7 & 5,5 \\
NO SUS & 16 & 50 & 39,7 \\
& 20 & 29 & 23,0 \\
\hline & 30 & $\mathbf{1 2 6}$ & $\mathbf{1 0 0 , 0}$ \\
\hline
\end{tabular}

Dos 97 Cirurgiões-Dentistas que realizaram a capacitação e continuam atendendo pelo SUS, 68 afirmam atender pacientes especiais. Os principais motivos para o não atendimento apontado pelos demais foram: ausência de incentivo por parte dos gestores para a implantação de um programa para atendimento de PNE $(n=10$ respostas); não há equipe auxiliar capacitada por isso não tem como trabalhar com os PNE (7 respostas); não se sente preparado(a), tendo apenas a capacitação, para o atendimento dos PNE (4 respostas); o atendimento muitas vezes é pré agendado nas unidades básica de saúde não deixando tempo para atender aos PNE (3 respostas); passou por uma experiência ruim no atendimento aos PNE e desistiu de atendê-los (2 respostas); trocou de cidade/concurso, e não conseguiu implementar o atendimento (2 respostas); não atende mais na parte clínica do SUS, trabalhando apenas na parte administrativa/gestão(2 respostas); não atende por que no seu município tem CEO e os PNE são atendidos lá (1 resposta)

Dos 80 Cirurgiões-Dentistas que trabalham em clínicas/ consultórios particulares, 46 afirmam atenderem pacientes com necessidades especiais nestes estabelecimentos, enquanto que 33 não os atendem e um não respondeu.

Além disso, os demais 10 que não exercem mais a odontologia afirmaram os seguintes motivos: aposentadoria (5 respostas), troca de profissão (3 respostas) e não trabalhar mais com odontologia devido a baixa remuneração (2 respostas).

De acordo com os CD, dos 496 municípios do Rio Grande do Sul, apenas $58(11,7 \%)$ possuem um ou mais CD capacitados que passaram pelo referido curso. Para melhor visualizar a distribuição e o percentual dos municípios dentro do Estado e, com isso, melhor compreender a abrangência do programa proposto pelo curso, decidiu-se utilizar as mesorregiões do Rio Grande do Sul (Tabela 2). 
Tabela 2 - Distribuição dos municípios com CD capacitados no RS.

\begin{tabular}{|c|c|c|c|}
\hline Mesorregião & $\begin{array}{c}\text { Total de } \\
\text { municípios }\end{array}$ & $\begin{array}{c}\text { Abrangência } \\
\text { n (\%) }\end{array}$ & Municípios com CD capacitado \\
\hline $\begin{array}{l}\text { Centro } \\
\text { Ocidental Rio- } \\
\text { grandense }\end{array}$ & 31 & $3(9,7)$ & Agudo; Santa Maria; São Sepé \\
\hline $\begin{array}{l}\text { Centro } \\
\text { Oriental Rio- } \\
\text { grandense }\end{array}$ & 54 & $9(16,7)$ & $\begin{array}{c}\text { Arroio do Tigre; Cerro Branco; } \\
\text { Lajeado; Pantano Grande; } \\
\text { Paverama; Rio Pardo; Santa } \\
\text { Cruz do Sul; Segredo; Venâncio } \\
\text { Aires }\end{array}$ \\
\hline $\begin{array}{l}\text { Metropolitana } \\
\text { de Porto } \\
\text { Alegre }\end{array}$ & 98 & $23(23,5)$ & $\begin{array}{c}\text { Alvorada; Barão do Triunfo; } \\
\text { Barra do Ribeiro; Cachoeirinha; } \\
\text { Canela; Canoas; Camaquã; } \\
\text { Campo Bom; Cerro Grande do } \\
\text { Sul; Esteio; Gramado; Gravataí; } \\
\text { Guaíba; Igrejinha; Ivoti; Novo } \\
\text { Hamburgo; Osório; Parobé; Porto } \\
\text { Alegre; Santa Maria do Herval; } \\
\text { São Leopoldo; Sentinela do Sul; } \\
\text { Torres }\end{array}$ \\
\hline $\begin{array}{l}\text { Nordeste Rio- } \\
\text { grandense }\end{array}$ & 53 & $7(13,2)$ & $\begin{array}{l}\text { Antônio Prado; Arvorezinha; } \\
\text { Caxias do Sul; Dois Lajeados; } \\
\text { São Jorge; São Marcos; Vacaria }\end{array}$ \\
\hline $\begin{array}{l}\text { Noroeste Rio- } \\
\text { grandense }\end{array}$ & 216 & $9(4,2)$ & $\begin{array}{l}\text { Alecrim; Ibiaçá; Novo Xingu; } \\
\text { Panambi; Rondinha; Santa Rosa; } \\
\text { Santo Ângelo; Santo Antônio do } \\
\text { Planalto; Três Passos }\end{array}$ \\
\hline $\begin{array}{l}\text { Sudeste Rio- } \\
\text { grandense }\end{array}$ & 25 & $4(16,0)$ & $\begin{array}{l}\text { Canguçu; Rio Grande; Santana } \\
\text { da Boa Vista; São Lourenço do } \\
\text { Sul }\end{array}$ \\
\hline $\begin{array}{l}\text { Sudoeste Rio- } \\
\text { grandense }\end{array}$ & 19 & $3(15,8)$ & $\begin{array}{l}\text { Alegrete; Rosário do Sul; } \\
\text { Uruguaiana }\end{array}$ \\
\hline Total & 496 & $58(11,7)$ & \\
\hline
\end{tabular}

\section{Discussão}

Muitos são os fatores que prejudicam o tratamento odontológico dos PNE tais como: situação socioeconômica, necessidade de grandes deslocamentos, dificuldade de transporte, bem como o tempo despedido em tratamentos de reabilitação paralelos ao tratamento odontológico (SILVA; LOBÃO, 2010; GUIMARÃES; AZEVEDO; SOLANO, 2006).

Aliado a isso, a literatura ainda aponta em diversos estudos nacionais (CARDOSO et al.. 2011a; PEREIRA et al. 2010; GUERREIRO; GARCIAS, 2009) e internacionais(CHI; ETTINGER, 2014; DEAL et al., 2004) uma dificuldade no acesso aos serviços odontológicos oferecidos a estes paciente, além do escasso número de profissionais dispostos a tratar estes pacientes (MOURADIAN; CORBIN, 2003), e outros que não se sentem capacitados ao atendimento (JUNG, 2011). Este quadro remete a uma alta prevalência de doenças bucais (LUCENA, 2013; JUNG, 2011; PEREIRA et al. 2010; ASA, 2007)

Buscando a melhora na saúde desses pacientes, a FADERS, em parceria com a ESP, realizava anualmente o 'Curso de Capacitação para Cirurgiões Dentistas e Pessoal Auxiliar em Odontologia para Atendimento à Pessoa com Deficiência e Necessidades Especiais', que, ao longo das dez primeiras edições, foi responsável pela capacitação de 145 cirurgiões dentistas. Destes 126 CDs responderam ao questionário desta pesquisa, sendo que apenas 68 continuaram a atender a PNEs no SUS, em 58 municípios do Estado do Rio Grande do Sul.

A grande maioria dos pacientes com necessidades especiais deveriam ter o seu atendimento odontológico solucionado no âmbito da atenção primária (BRASIL, 2008) por CD habilitados ao atendimento e, ao constatar impossibilidade da prestação de serviço nesse nível de atenção, o paciente seria encaminhado para 0 atendimento de referência nos Centros de Especialidades Odontológicas(FRAZÃO; NARVAI, 2009).

Apesar disso, a formação do CD no que diz respeito à atenção odontológica aos pacientes com necessidades especiais ainda é falha (VARELLIS, 2005). Sendo necessário estratégias que capacitem o acadêmico no exercício e na elaboração de planejamentos em saúde para os diferentes tipos populacionais(VOLPATO et al., 2014), além de fomentar cursos de pós graduação que desenvolvam capacidades e competências no cuidado dessas populações especiais (JUNG, 2011).

Por meio do Sistema Único de Saúde, torna-se necessário pensar no processo de trabalho em que os trabalhadores possam desempenhar eficientemente suas funções, sendo valorizados por vínculos empregatícios fortes à instituição, remunerações adequadas, e número de recursos humanos suficientes e capacitados, para assim propiciar motivação e segurança nos profissionais, a fim de fornecer um atendimento de qualidade que de continuidade nas ações propostas, e com um cuidado adequado a saúde bucal destes pacientes (SANTANA, 2001).

O papel dos gestores deve ser mais amplo e comprometido com a efetivação de políticas públicas voltadas a saúde das pessoas com necessidades especiais (VANDERLEI; ALMEIDA, 2007). Faz-se necessária uma melhor avaliação e distribuição, dos serviços especializados da atenção secundária e terciária ao longo do Estado, visando atender aqueles pacientes que realmente necessitem de assistência especializada de forma rápida, segura e eficaz, sem onerar o serviço com atendimentos que poderiam ser realizados no primeiro nível de atenção(ALVES, 2011).

Espera-se que os resultados apresentados aqui possam subsidiar pesquisadores, gestores e profissionais do Estado do Rio Grande do Sul, na elaboração e implementação de políticas públicas cujo objetivo seja melhorar o cuidado prestado pelos serviços de saúde às pessoas portadoras de necessidades especiais, a fim de assegurar a igualdade de oportunidades no atendimento. Ademais, sugere-se que as discussões sobre a saúde da pessoa com deficiência, relacionada aos serviços de saúde, ocorra ela dentro ou fora do contexto do Sistema Único de Saúde

Algumas limitações são inerentes ao estudo. Dado o seu desenho transversal, o estudo foi limitado à identificação de exploração ao invés de relações causais. Ainda, a presença de outros fatores contextuais, inerentes ao curso e ao perfil dos cirurgiõesdentistas estudados, e não contemplados na análise, devem ser investigados com maior profundidade.

Por fim, torna-se necessário examinar permanentemente as políticas voltadas a saúde bucal dos pacientes com necessidades especiais, uma vez que percebeu-se a necessidade de ampliação de acesso aos serviços e no tratamento desses pacientes, além do desenvolvimento de capacitações e especializações aos cirurgiões dentistas para o cuidado desses pacientes.

\section{Conclusão}

Os resultados do presente estudo demonstraram o perfil dos participantes que participaram do Curso de Capacitação para Cirurgiões Dentistas no Atendimento à Pessoa com Deficiência e Necessidades Especiais do Estado do Rio Grande do Sul, além do seu percurso no atendimento a esses pacientes e a cobertura dos municípios beneficiados com o programa.

O entendimento dos recursos humanos disponíveis para o Estado, seu vínculo empregatício, e capacitações para o atendimento, é fundamental para uma melhor avaliação e distribuição dos serviços de saúde bucal voltado a essa população, a fim de assegurar a igualdade de oportunidades, e com um cuidado adequado a saúde bucal destes pacientes. Ademais, faz-se necessária uma avaliação permanente, dos serviços ofertados pelo 
Estado do Rio Grande do Sul e das políticas voltadas a saúde bucal dos pacientes com necessidades especiais.

Os principais motivos apontados para o não atendimento dessa população foram: não houve incentivo por parte dos gestores para a implantação de um programa para atendimento de pacientes especiais, não há equipe auxiliar capacitada e não se sente preparado, tendo apenas a capacitação, para o atendimento dos PNE. A partir dessa exploração inicial, em estudos futuros, uma análise em maior profundidade deve contemplar esses fatores.

\section{Referências}

ALVES, J.S.; ASSIS M.M.A. Gestão do trabalho: abordagens teóricas e políticas no contexto do sistema único de saúde (sus). Rev. Baiana de Saúde Pública.v.35, n.1, p.55-67, jan./jun. 2011.

ASA, R. Treating patients with special needs requires both training and compassion. AGD Impact. v.35, n.10, p.34-8, 2007.

BRASIL, Ministério da Saúde. Secretaria de Atenção à Saúde. Departamento de Atenção Básica. Coordenação Nacional de Saúde Bucal. Cadernos de atenção básica. Saúde Bucal n¹7. Brasília: MS; 2008.

CARDOSO, A.M.R.; BRITO, D.B.A.; ALVES, V.F.; PADILHAW.W.N. O Acesso ao Cuidado em Saúde Bucal para Crianças com Deficiência Motora: Perspectivas dos Cuidadores. Pesq Bras Odontoped Clin Intergr, João Pessoa, v.11, n.4, p.593-99, out./dez. 2011a.

CHI, D.L.; ETTINGER, R.L. Prevention and nonsurgical management of dental caries over the life course for individuals

with special healthcare needs. J Calif Dent Assoc. v.42, n.7, p.45563, jul. 2014.

CINGANO, L.; AONZO, E.; SERVETTO, R.; CALCAGNO, E. Aspects related to a periodontal home prevention program for disabled patients: a clinical trial. Minerva Stomatologica. v.62, n.12, p.419-30, nov./dez. 2013.

CONSELHO FEDERAL DE ODONTOLOGIA. Aprova a Consolidação das Normas para Procedimentos nos Conselhos de Odontologia.Resolução do Conselho Federal de Odontologia n. ${ }^{\circ}$ CFO-63, de 20 de maio de 2005, atualizada em junho de 2012. Lex:

Consolidação das normas para procedimentos nos conselhos de odontologia, Brasília, p. 01-86, jun de 2012. Legislação Federal e Marginália.

\section{CONSELHO FEDERAL DE ODONTOLOGIA. Dados Estatísticos.}

Brasília - DF. Disponível em: <http://cfo.org.br/servicos-econsultas/Dados-

estatisticos/?elemento=especialistas\&especialidade=21\&cro=RS\&mu nicipio=> Acesso em: 29 dez. 2014.

DEAL, A.; ROSEMAN, J.; PASS, M.A.; THORNTON, J.B.; CHAVERS, L.S. Access to dental care in alabama for children with special needs. J Am Dent Assoc. v.135, n.4, p490-5, 2004.

FRAZÃO, P.; NARVAI, P.C. Saúde bucal no Sistema Único de Saúde: 20 anos de lutas por uma política pública. Saúde em debate, Rio de Janeiro, v.33, n.81, jan./abr. 2009.

GUERREIRO, P.O.; GARCIAS, G.L. Diagnóstico das condições de saúde bucal em portadores de paralisia cerebral do município de
Pelotas, Rio Grande do Sul, Brasil. Ciência \& Saúde Coletiva, v.14, n.5, p.1939-46, 2009.

GUIMARÃES, A.O.; AZEVEDO, I.D.; SOLANO, M.C. Medidas preventivas em odontologia para pacientes portadores de necessidades especiais. Rev. Ibero-Am Odontopediatr Odontol Bebê. v.9, n.47, p.79-84, 2006.

JUNG, L.S. Serviços odontológicos oferecidos às crianças com necessidades especiais. 2011. 37f. Trabalho de conclusão (Especialização em saúde pública) - Faculdade de Medicina, Universidade Federal do Rio do Sul, Porto Alegre.

LUCENA, C.D.R.X.; LUCENA, E.H.G.; SOUSA, M.F.; MENDONÇA A.V.M. Análise dos atendimentos odontológicos em pessoas com necessidades especiais em modalidade hospitalar no Sistema Único de Saúde, Brasil, 2012. Tempus Actas de Saúde Coletiva, [S.I.], v. 7, n. 2, p. 173-82, set. 2013.

MOURADIAN, W.; CORBIN, S. Addressing health disparities through dental medical collaborations, part II. Cross-cutting themes in the care of special populations. Journal of Dental Education, Seattle, v.67, n.2, p.1320-26, dez. 2013.

PEREIRA, L.M.; MARDERO, E.; FERREIRA, S.H.; KRAMER, P.F.; COGO, R.B. Atenção odontológica em pacientes com deficiências: a experiência do curso de Odontologia da ULBRA. Stomatos, Canoas, v. 16, n. 31, p. 92-99, jul./dez. 2010.

POLIT, D.F.; BECK C.T.; HUNGLER B.P. Fundamentos de pesquisa em enfermagem: métodos, avaliação e utilização. $5^{a}$ ed. Porto Alegre: Artmed; 2004.

SANTOS, M.T.; GUARÉ, R. O.; LEITE, M. F.; FERREIRA, M.C.; DURÃO, M.S.; JARDIM, J.R. Salivary osmolality in individuals with cerebral palsy. Arch Oral Biol. v.55, n.11, p.855-60, 2010a.

SANTOS, M.T.; GUARÉ, R.O.; LEITE, M.F.; FERREIRA, M.C.; NICOLAU, J. Does the neuromotor abnormality type affect the salivary parameters in individuals with cerebral palsy? J Oral Biol Med. v.39, n.10, p.770-4, 2010b.

SANTOS, M.T.; NOGUEIRA M.L. Infantile reflexes and their effects on dental caries and oral hygiene in cerebral palsy individuals. J Oral Rehabil. v.32, n.12, p.880-5, 2005.

SILVA, L.C.P.; LOBÃO, D.S. Manejo de Pacientes com Necessidades Especiais nos cuidados de saúde. In. MASSARA, M.L.A.; RÉDUA, P.C. Manual de referência para procedimentos clínicos em odontopediatria. São Paulo: Santos; p.263-72, 2010.

SILVESTRE, F.J.; PLAZA, A. Odontología en pacientes especiales. Valencia: Universidad de Valencia PUV, 2007. 314p.

TANAKA, M.H.; BOCARDI, K.; KISHIMOTO, K.Y.; JACQUES, P; SPOLIDORIO, D.M.P.; GIRO, E.M.A. DMFT index assessment and microbiological analysis of Streptococcus mutans in institutionalized patients with special needs. Braz J Oral Sci. v.8, n.1, p.9-13, 2009.

TOBAR, F; YALOUR, M.R. Como fazer teses em saúde publica: conselhos e ideias para formular projetos e redigir teses e informes de pesquisa. Rio de Janeiro: Fiocruz, 2001.

VANDERLEI, M. I. G.; ALMEIDA, M. C. P. A concepção e prática dos gestores e gerentes da estratégia de saúde da família. Ciência \& 
Saúde Coletiva, Rio de Janeiro, v. 12, n. 2, p. 443-454, mar./abr. 2007.

VARELLIS, M.L.Z. O paciente com necessidades especiais na odontologia: manual prático. São Paulo: Santos; 2005.

VOLPATO, S.; GALLON, A.; DÉA, B.E.; TOMASI, P.Z.; MAKOWSKI, R.M.;

MANFRO, A.R.G.; GARRASTAZU, M.D. A construção do conhecimento pelo acadêmico de odontologia sobre paciente especial: relato de experiência. Ação odonto, v.2, n.1, p.47-59, 2014. 